\title{
A note on regular Ramsey graphs
}

\author{
Noga Alon* $\quad$ Sonny Ben-Shimon ${ }^{\dagger} \quad$ Michael Krivelevich ${ }^{\ddagger}$
}

November 9, 2018

\begin{abstract}
We prove that there is an absolute constant $C>0$ so that for every natural $n$ there exists a trianglefree regular graph with no independent set of size at least $C \sqrt{n \log n}$.
\end{abstract}

\section{Introduction}

A major problem in extremal combinatorics asks to determine the maximal $n$ for which there exists a graph $G$ on $n$ vertices such that $G$ contains no triangles and no independent set of size $t$. This Ramsey-type problem was settled asymptotically by Kim [6] in 1995, after a long line of research; Kim showed that $n=\Theta\left(t^{2} / \log t\right)$. Recently, Bohman [1] gave an alternative proof of Kim's result by analyzing the so-called triangle-free process, as proposed by Erdős, Suen and Winkler [3], which is a natural way of generating a triangle-free graph. Consider now the above problem with the additional constraint that $G$ must be regular. In this short note we show that the same asymptotic results hold up to constant factors. The main ingredient of the proof is a gadget-like construction that transforms a triangle-free graph with no independent set of size $t$, which is not too far from being regular, into a triangle-free regular graph with no independent set of size $2 t$.

Our main result can be stated as follows.

Theorem 1.1. There is a positive constant $C$ so that for every natural $n$ there exists a regular triangle-free graph $G$ on $n$ vertices whose independence number satisfies $\alpha(G) \leq C \sqrt{n \log n}$.

Denote by $R(k, \ell)$ the maximal $n$ for which there exists a graph on $n$ vertices which contains neither a complete subgraph on $k$ vertices nor an independent set on $\ell$ vertices. Let $R^{\text {reg }}(k, \ell)$ denote the maximal $n$ for which there exists a regular graph on $n$ vertices which contains neither a complete subgraph on $k$ vertices

*School of Computer Science and School of Mathematical Sciences, Raymond and Beverly Sackler Faculty of Exact Sciences, Tel Aviv University, Tel Aviv 69978, Israel. E-mail: nogaa@post.tau.ac.il. Research supported in part by an ERC advanced grant, by the Israel Science Foundation and by a USA-Israel BSF grant.

†School of Computer Science, Raymond and Beverly Sackler Faculty of Exact Sciences, Tel Aviv University, Tel Aviv 69978, Israel. E-mail: sonny@post.tau.ac.il. Research conducted as part of the author's Ph.D. thesis under the supervision of Prof. Michael Krivelevich.

${ }^{\ddagger}$ School of Mathematical Sciences, Raymond and Beverly Sackler Faculty of Exact Sciences, Tel Aviv University, Tel Aviv 69978, Israel. E-mail: krivelev@post.tau.ac.il. Research supported in part by USA-Israel BSF Grant 2006322, by grant 1063/08 from the Israel Science Foundation, and by a Pazy memorial award. 
nor an independent set on $\ell$ vertices. Clearly, for every $k$ and $\ell$ one has $R^{\text {reg }}(k, \ell) \leq R(k, \ell)$. Theorem 1.1 states that $R^{\mathrm{reg}}(3, t)=\Theta(R(3, t))=\Theta\left(\frac{t^{2}}{\log t}\right)$.

\section{Proof of Theorem 1.1}

Note first that the statement of the theorem is trivial for small values of $n$. Indeed, for every $n_{0}$ one can choose the constant $C$ in the theorem so that for $n \leq n_{0}, C \sqrt{n \log n} \geq n$, implying that for such values of $n$ a graph with no edges satisfies the assertion of the theorem. We thus may and will assume, whenever this is needed during the proof, that $n$ is sufficiently large.

The following well known theorem due to Gale and to Ryser gives a necessary and sufficient condition for two lists of non-negative integers to be the degree sequences of the classes of vertices of a simple bipartite graph. The proof follows easily from the max-flow-min cut condition on the appropriate network flow graph (see e.g. [8, Theorem 4.3.18]).

Theorem 2.1 (Gale; Ryser). If $\mathbf{d}=\left(d_{1}, \ldots, d_{m}\right)$ and $\mathbf{d}^{\prime}=\left(d_{1}^{\prime}, \ldots, d_{n}^{\prime}\right)$ are lists of non-negative integers with $d_{1} \geq \ldots \geq d_{m}, d_{1}^{\prime} \geq \ldots \geq d_{n}^{\prime}$ and $\sum d_{i}=\sum d_{j}^{\prime}$ then there exists a simple bipartite graph with degree sequences $\mathbf{d}$ and $\mathbf{d}^{\prime}$ on each side respectively iff $\sum_{i=1}^{m} \min \left\{d_{i}, s\right\} \geq \sum_{j=1}^{s} d_{j}^{\prime}$ for every $1 \leq s \leq n$.

Corollary 2.2. Let $a \geq 1$ be a real. If $\mathbf{d}=\left(d_{1}, \ldots, d_{m}\right)$ is a list of non-negative integers with $d_{1} \geq \ldots \geq d_{m}$ and

$$
d_{1} \leq \min \left\{a d_{m}, \frac{4 a m}{(a+1)^{2}}\right\}
$$

then there exists a simple bipartite graph with degree sequence $\mathbf{d}$ on each side. In particular, this holds for $d_{1} \leq \min \left\{2 d_{m}, \frac{8 m}{9}\right\}$.

Proof. By Theorem 2.1]it suffices to check that for every $s, 1 \leq s \leq m, \sum_{i=1}^{s} d_{i} \leq \sum_{i=1}^{m} \min \left\{d_{i}, s\right\}$. Suppose this is not the case and there is some $s$ as above so that

$$
d_{1}+d_{2}+\ldots+d_{s}>\sum_{i=1}^{m} \min \left\{d_{i}, s\right\}
$$

If $d_{i}<d_{1}$ for some $i$ satisfying $2 \leq i \leq s$, replace $d_{i}$ by $d_{1}$. Observe that by doing so the left hand side of (2) increases by $d_{1}-d_{i}$, whereas the right hand side increases by at most this quantity, hence (2) still holds with this new value of $d_{i}$. We can thus assume that $d_{1}=d_{2}=\cdots=d_{s}$. Note that if $d_{1} \leq s$, then (2) cannot hold, hence $d_{1}>s$. If $d_{i}>d_{1} / a$ for some $i$ satisfying $s+1 \leq i \leq m$, then reducing it to $d_{1} / a$ (even if this is not an integer), maintains (2), as the left hand side does not change, whereas the right hand side can only decrease. Moreover, the new sequence still satisfies (11). Thus we may assume that in (2) $d_{i}=d_{1} / a$ for all $s+1 \leq i \leq m$. Put $d=d_{i+1}\left(=d_{i+2}=\ldots=d_{m}\right)$, then (2) gives

$$
d_{1}+\ldots+d_{s}=s \cdot(a d)>\sum_{i=1}^{m} \min \left\{d_{i}, s\right\}=s^{2}+(m-s) d .
$$

Therefore $[(a+1) s-m] d>s^{2}$, implying that $(a+1) s-m>0$, that is, $s>\frac{m}{a+1}$, and

$$
d>\frac{s^{2}}{(a+1) s-m} .
$$


The function $g(s)=\frac{s^{2}}{(a+1) s-m}$ attains its minimum in the range $\frac{m}{a+1}<s \leq m$ at $s=\frac{2 m}{a+1}$ and its value at this point is $\frac{4 m}{(a+1)^{2}}$. We thus conclude from (3) that $d>\frac{4 m}{(a+1)^{2}}$ and hence that $d_{1}=a d>\frac{4 a m}{(a+1)^{2}}$ contradicting the assumption (11). This completes the proof.

Remark 2.3. Condition (11) is tight for all values of $a>1$, in the sense that if $d_{1}>\frac{4 a m}{(a+1)^{2}}$ and $d_{1}=d_{2} \ldots=$ $d_{s}$ for $s=\frac{2 m}{a+1}$ with $d_{i}=\frac{4 m}{(a+1)^{2}}$ for all $s+1 \leq i \leq m$, then there is no simple bipartite graph whose degree sequence in each side is $\left(d_{1}, d_{2}, \ldots, d_{m}\right)$. This follows from Theorem 2.1.

Let $\mathcal{R}(n, 3, t)$ denote the set of all triangle-free graphs $G$ on $n$ vertices with $\alpha(G)<t$. As usual, let $\Delta(G)$ and $\delta(G)$ denote the respective maximal and minimal degrees of $G$.

Proposition 2.4. Let $t$ and $d$ be integers. If there exists a graph $G \in \mathcal{R}(n, 3, t)$ such that $\Delta(G)-\delta(G) \leq$ $d \leq \frac{4}{9} \cdot\left\lfloor\frac{n}{\Delta(G)+1}\right\rfloor$, then there exists a $(d+\Delta(G))$-regular graph $G^{\prime} \in \mathcal{R}(2 n, 3,2 t-1)$.

Proof. Construct a new graph $G^{\prime}$ as follows. Take two copies of $G$, and color each of these copies by the same equitable coloring using $\Delta(G)+1$ colors with all color classes of cardinality either $\lfloor n /(\Delta(G)+1)\rfloor$ or $\lceil n /(\Delta(G)+1)\rceil$ using the Hajnal-Szemerédi Theorem [4] (see also a shorter proof due to Kierstead and Kostochka [5]). Let $C$ and $C^{\prime}$ be the same color class in each of the copies of $G$. Denote the degree sequence of the vertices of $C$ in $G$ by $d_{1}^{\prime} \leq \ldots \leq d_{m}^{\prime}$, where $m=|C|$, and set $d_{i}=d+\Delta(G)-d_{i}^{\prime}$. According to Corollary 2.2 there exists a simple bipartite graph with $m$ vertices on each side, where the degree sequence of each side is $d_{1} \geq \ldots \geq d_{m}$ as the maximal degree $d_{1}=d+\Delta(G)-\delta(G) \leq 2 d$, the minimal degree $d_{m} \geq d$, and by our assumption on $G$ we have $d_{1} \leq \frac{8 m}{9}$. We can thus connect the vertices of $C$ and $C^{\prime}$ using this bipartite graph such that all vertices in $C \cup C^{\prime}$ have degree $d+\Delta(G)$. By following this method for every color class, we create the graph $G^{\prime}$ which is $(d+\Delta(G))$-regular, triangle-free and has no independent set of cardinality $2 t-1$.

\subsection{The $H$-free process and Bohman's result}

Consider the following randomized greedy algorithm to generate a graph on $n$ labeled vertices with no $H$ subgraph for some fixed graph $H$. Given a set of $n$ vertices, a sequence of graphs $\left\{G_{i}^{(H)}\right\}_{i=0}^{t}$ on this set of vertices is constructed. Start with $G_{0}^{(H)}$ as the empty graph, and for each $0<i \leq t$, the graph $G_{i}^{(H)}$ is defined by $G_{i-1}^{(H)} \cup\left\{e_{i}\right\}$ where $e_{i}$ is chosen uniformly at random from all unselected pairs of vertices that do not create a copy of $H$ when added to $G_{i-1}^{(H)}$. The process terminates at step $t$, the first time that no potential unselected pair $e_{t+1}$ exists. This algorithm is called the $H$-free process.

The $K_{3}$-free process was proposed by Erdős, Suen and Winkler [3] and was further analyzed by Spencer [7. Recently, Bohman [1] extending and improving previous results, was able to analyze the $K_{3}$-free process and to show that with high probability it passes through an almost regular Ramsey-type graph.

Theorem 2.5 (Bohman [1]). With high probability 1 there exists an integer $1 \leq m=m(n)$ such that the following properties hold simultaneously:

1. $G_{m}^{\left(K_{3}\right)} \in \mathcal{R}(n, 3, C \sqrt{n \log n})$ for some absolute constant $C>0$;

2. $\Delta\left(G_{m}^{\left(K_{3}\right)}\right)=\Theta(\sqrt{n \log n})$;

\footnotetext{
${ }^{1}$ In this context we mean that the mentioned events hold with probability tending to 1 as $n$, the number of vertices, goes to infinity.
} 
3. $\Delta\left(G_{m}^{\left(K_{3}\right)}\right)-\delta\left(G_{m}^{\left(K_{3}\right)}\right)=o(\sqrt{n / \log n})$.

Remark 2.6. Item (3) can be derived implicitly from [1], or alternatively, it follows from [2, Theorem 1.4], as the degree of every vertex is a trackable extension variable.

Note that Proposition 2.4 in conjunction with Theorem 2.5 completes the proof of Theorem 1.1 for every large enough even integer $n$. To fully complete the proof, we describe how to deal with the case of $n$ odd. So, let now $n$ be be large enough and odd. Our aim is to show the existence of a regular triangle-free graph $G_{n}$ on $n$ vertices with $\alpha\left(G_{n}\right)=O(\sqrt{n \log n})$. The approach we take to achieve this goal is to construct a "big" graph satisfying our Ramsey conditions on an even number of vertices, and to add to it a "small" graph with an odd number of vertices without affecting the asymptotic results claimed.

For every $k=0(\bmod 5)$, and every even $r \leq 2 k / 5$, let $H_{k, r}$ denote a graph constructed as follows. Start with a copy of $C_{5}$ blown up by factor of $k / 5$ and delete from the resulting graph $(2 k / 5-r / 2)$ disjoint 2-factors (which exist by Petersen's Theorem, see e.g. [8, Theorem 3.3.9]). $H_{k, r}$ is hence a triangle-free $r$-regular graph on $k$ vertices.

Denote by $F_{n}$ an $r$-regular triangle-free graph on $2 n$ vertices with $\alpha\left(F_{n}\right) \leq C \sqrt{n \log n}$ for some absolute constant $C$, and furthermore assume $r$ is even (this can be achieved by choosing the appropriate parameter $d$ in Proposition 2.4, as we have much room to spare with the values we plug in from Theorem 2.5). Let $n_{0}=(n-k) / 2$, where $k=5(\bmod 10)$, and $k=(1+o(1)) \frac{5 C}{2} \sqrt{n \log n}$. Clearly, $n_{0}$ is integer. The graph $F_{n_{0}}$ is $r$-regular for some even $r \leq \alpha\left(F_{n_{0}}\right)$, is triangle-free on $2 n_{0}$ vertices, and satisfies $\alpha\left(F_{n_{0}}\right) \leq C \sqrt{n_{0} \log n_{0}} \leq$ $C \sqrt{n \log n}$. Now, define $G_{n}$ to be a disjoint union of $F_{n_{0}}$ and $H_{k, r}$. Clearly, $G_{n}$ has $2 n_{0}+k=n$ vertices, is $r$-regular, triangle-free and satisfies $\alpha\left(G_{n}\right)=\alpha\left(F_{n_{0}}\right)+\alpha\left(H_{k, r}\right) \leq \alpha\left(F_{n_{0}}\right)+k \leq C \sqrt{n \log n}+k=O(\sqrt{n \log n})$.

\section{Discussion}

A natural question that extends the above is to try and determine $R^{\text {reg }}(k, \ell)$ for other values of $k$ and $\ell$ (in particular for fixed values of $k>3$ and $\ell$ arbitrary large), and also to try and investigate its relation with $R(k, \ell)$. The following conjecture seems plausible.

Conjecture 3.1. For every $k \geq 2$ there is a constant $c_{k}>0$ so that $R^{\text {reg }}(k, \ell) \geq c_{k} R(k, \ell)$ for all $\ell \geq 2$.

This is trivial for $k=2$, and by our main result here holds for $k=3$ as well.

Recently, Bohman and Keevash [2] were able to generalize the techniques of [1] for the $H$-free process, where $H$ is a strictly 2-balanced graph. This in turn provided new lower bounds for $R(k, \ell)$ (as complete graphs are strictly 2-balanced) where $k$ is fixed and $\ell$ arbitrarily large. It is plausible to think that these results can also be used to construct regular Ramsey graphs in a manner similar to that described in this

note. Nonetheless, since the asymptotic behavior of $R(k, \ell)$ is not known for $k \geq 4$, a complete proof of Conjecture 3.1 appears to require some additional ideas, and remains open.

\section{References}

[1] T. Bohman. The triangle-free process. Advances in Mathematics, 221(5):1653-1677, 2009. 
[2] T. Bohman and P. Keevash. The early evolution of the H-free process. Manuscript.

[3] P. Erdős, S. Suen, and P. Winkler. On the size of a random maximal graph. Random Structures and Algorithms, 6(2-3):309-318, 1995.

[4] A. Hajnal and E. Szemerédi. Proof of a conjecture of Erdős. In P. Erdős, A. Rényi, and V. T. Sòs, editors, Combinatorial Theory and Its Applications, volume 2, pages 601-623. Amsterdam, Netherlands: North-Holland, 1970.

[5] H. A. Kierstead and A. V. Kostochka. A short proof of the Hajnal-Szemerédi theorem on equitable colouring. Combinatorics, Probability and Computing, 17(2):265-270, 2008.

[6] J. H. Kim. The Ramsey number $R(3, t)$ has order of magnitude $\frac{t^{2}}{\log t}$. Random Structures and Algorithms, $7(3): 173-207,1995$.

[7] J. Spencer. Maximal triangle-free graphs and Ramsey $R(3, t)$. Unpublished manuscript, 1995.

[8] D. B. West. Introduction to Graph Theory. Prentice Hall, 2nd edition, 2001. 